\section{Gold Compounds in Medicine: Potential Anti-Tumour Agents}

\author{
Edward R.T. Tiekink \\ Department of Chemistry, National University of \\ Singapore, Singapore 117543; \\ E-mail: chmtert@nus.edu.sg
}

An overview of the use of gold drugs in the alleviation of the symptoms associated with the debilitating disease rheumatoid arthritis (RA) is presented. Other potential therapies based on gold compounds, such as against parasitic diseases, HIV and asthma are summarised. The development of gold compounds as novel anti-tumour agents is also described. Compounds containing gold(I), as utilised in the treatment of RA, and gold(III), show exciting potential in this regard, there being some very potent compounds targeting biological targets, such as DNA, and displaying selectivity in their cytotoxic profiles. In conclusion, very real potential exists for the development of anti-tumour agents based on gold.

\section{Introduction}

Regular readers of Gold Bulletin will be aware of the many and varied applications of gold and its compounds. One only has to look in the 'Literature Highlights' section of Gold Bulletin to note advances in catalysis, nanotechnology, electronics and medicine, to name but a few areas of active research. Of these, it is the last-named endeavour, i.e. the use of gold compounds in medicine, that forms the focus of this overview.

The use of gold for medicinal purposes, real or perceived, dates back to antiquity with Arabic, Indian and Chinese physicians prescribing gold preparations for the treatment of a variety of ailments; an historical perspective of the use of gold or gold compounds in medicine is available (1) as is a more recent survey of early Chinese use of gold (2). In more recent times, gold compounds find clinical use as antiarthritic agents, i.e. chrysotherapy, as discussed below (3). In addition, exploration continues into the potential use of gold in the treatment of cancer (4), as anti-HIV (5) and antimalarial agents (6), and for the treatment of bronchial asthma (7). The purpose of this review is to highlight the current use of gold compounds in medicine and to summarise current research directed towards developing gold-based anti-tumour drugs.

\section{Rheumatoid Arthritis and Treatments of the Disease}

Rheumatoid arthritis (RA) is one of over 100 arthritic diseases afflicting humans with other common forms being osteoarthritis and gout. RA accounts for about 5 - 10\% of all patients suffering arthritic diseases and is a particularly debilitating form of arthritis. Manifestations of RA include inflammation with concomitant restriction of movement and pain of joints such as wrists, hands and feet. While the disease can occur at any age, patients below 25 years are relatively rare although the onset of the disease may occur at an early age. There is no pattern of inheritance and females are afflicted to a greater extent than men in the approximate ratio 4:1. The aetiology of RA is not known although it is likely due to a breakdown of the immune system. A patient is usually diagnosed with RA if the following three factors are satisfied: i) obvious inflammation, ii) erosion of the joints and iii) presence of the rheumatoid factor associated with the production of immunoglobulin. In summary, RA is a relatively common and incapacitating disease with recognisable manifestations but, one that is not well understood. Besides the universal call to adopt better living standards, i.e. rest, regular exercise and good diet, chemotherapy is at the forefront of treatment. 
It should be stated from the outset that there is no cure for RA; medication serves to alleviate pain and/or halt the progression of the disease but, does not cure the disease. There are several classes of drugs employed in the control of RA. The first of these are Non-Steroidal Anti-Inflammatory Drugs (NSAIDS). These function by blocking the production of prostaglandins, the chemicals responsible for promoting pain and inflammation. Amongst the NSAIDS that may be familiar to readers are Advil $^{\circledR}$ (ibuprofen), Feldene ${ }^{\circledR}$ (piroxicam), Lodine ${ }^{\circledast}$ (etodolac), Naprosyn ${ }^{\circledR}$ (naproxen), and Voltaren ${ }^{\circledR}$ (diclofenac sodium), to name but a few examples. However, NSAIDS do not differentiate between the 'good' (i.e. prostaglandins that protect kidney function and the stomach lining) and 'bad' prostaglandins (that promote inflammation). Another relatively new class of drugs are the COX-\|l enzyme inhibitors such as Celebrex ${ }^{\circledR}$ (celecoxib), Mobic ${ }^{\circledR}$ (meloxicam) and Vioxx ${ }^{\circledR}$ (rofecoxib). These drugs have selectivity in their mode of action in that they target the inflammatory arthritis. The salicylates constitute another important class of drugs used to relieve the pain and inflammation associated with RA, of which Aspirin ${ }^{\circledR}$ is a notable example. Glucocorticoids are drugs based on the naturally occurring hormone, cortisone, and can rapidly act to reduce the pain and swelling but, can induce serious side-effects such as reducing resistance to infection, weight gain, loss of muscle strength, cataracts, diabetes, osteoporosis, etc. The final class of drugs to be briefly described, include gold compounds, and are termed Disease-Modifying Antirheumatic Drugs, the DMARDS.
As the name suggests, DMARDS, act to impede or even stop the progression of RA. These are appropriately employed for newly-diagnosed patients so as to manage the disease by halting progression. Examples of non-gold-based DMARDS ${\text { include } \text { Arava }^{\circledR} \text { (leflunomide), Azulfidine }}^{\circledast}$ (imuran), Cytoxan ${ }^{\circledR}$ (cyclophosphamide), Novo-chloroquine ${ }^{\circledR}$ (chloroquine), and Rheumatrex $^{\circledR}$ (methotrexate). While there are the inevitable side-effects associated with the above-named DMARDS, such as flu-like symptoms, these are the price a patient has to pay in order to retard the progression of the disease. As mentioned above, gold-based compounds are also classed as DMARDS, as there is experimental evidence to indicate that chrysotherapy is disease modifying. However, it is fair to say that gold-drugs are probably used more as a last-line of defence against RA. Why this is so is not totally clear. Perhaps this represents a general aversion to "heavy-metal therapy". As discussed below, there are side-effects associated with chrysotherapy but, this is true for all chemotherapies. Having stated the above, there are a number of gold-based drugs currently available for the treatment of the symptoms associated with RA.

\section{Chrysotherapy}

There are two classes of gold compounds used in the treatment of RA. Membership of each class relates to the inherent chemistry of the compound and dictates their

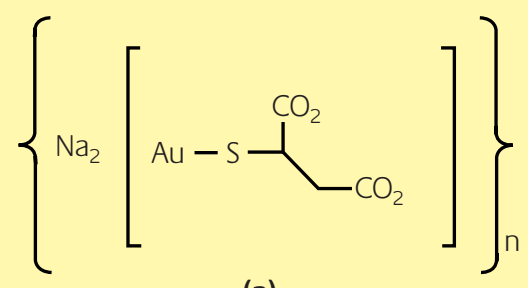

(a)

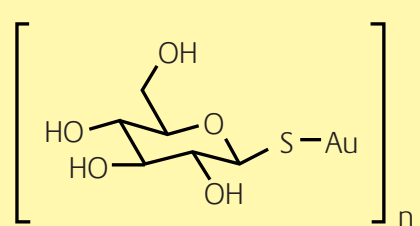

(b)

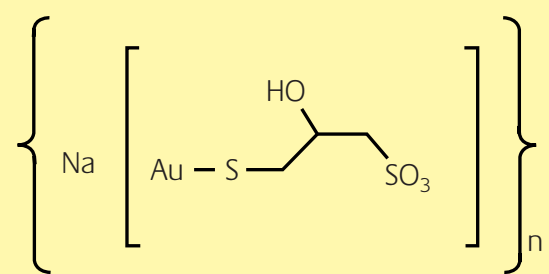

(c)

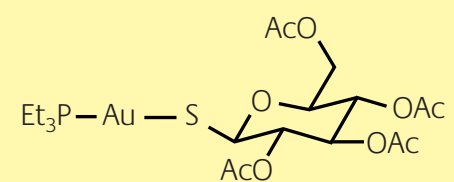

(e)

Figure 1

Chemical representations of (a) sodium aurothiomalate, (b) aurothioglucose, (c) sodium aurothiopropanol sulphonate, (d) sodium aurothiosulphate, and (e) auranofin 


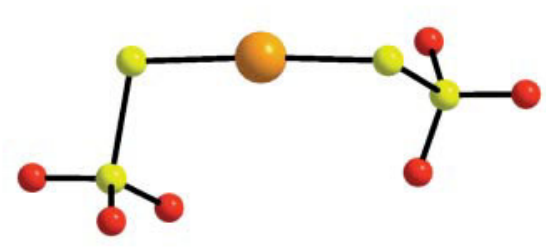

Figure 2

The molecular structure of the monomeric trianion found in the crystal structure of sodium aurothiosulphate. The diagram was drawn with DIAMOND ${ }^{\circledR}$ (9). Colour code: Gold, orange; sulphur, yellow; oxygen, red

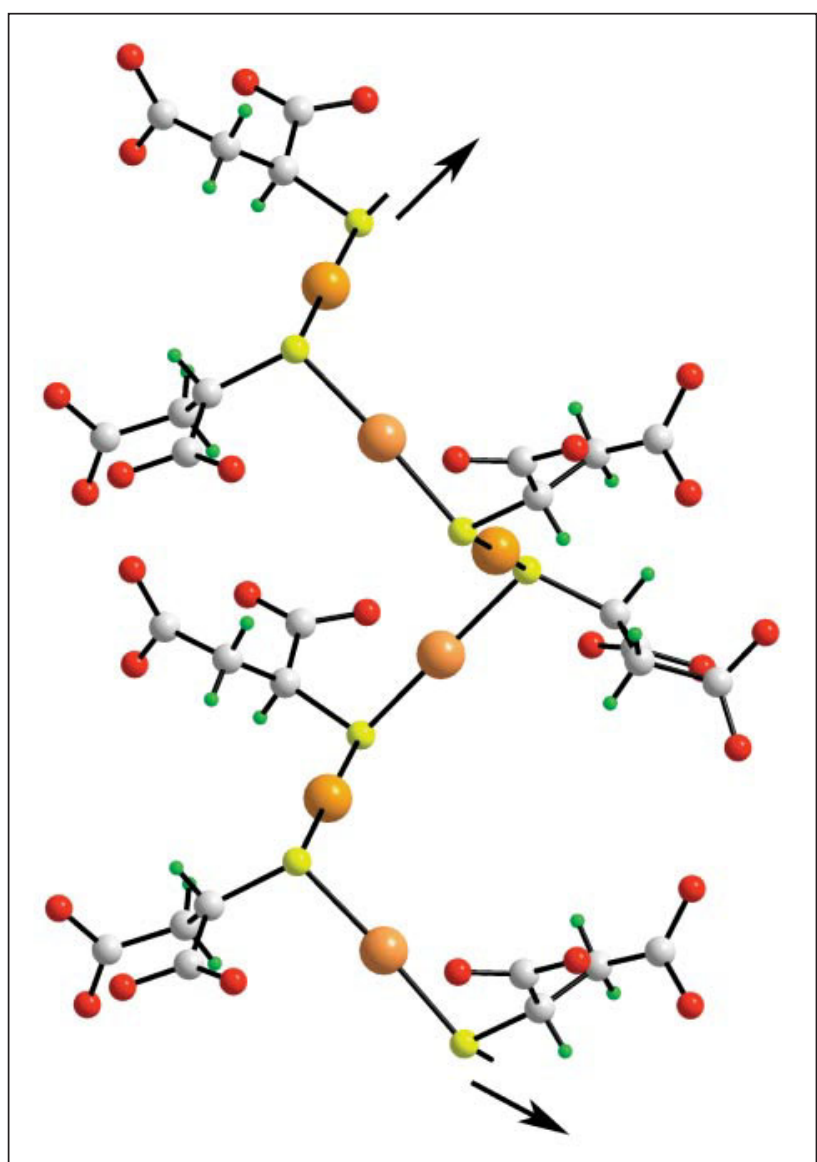

Figure 3

The molecular structure of the polymeric dianion found in the crystal structure of $\left[\mathrm{Na}_{2} \mathrm{CS}(\mathrm{H})\right]\left[\left\{\mathrm{Au}\left(\mathrm{SCH}\left(\mathrm{CO}_{2}\right) \mathrm{CH}_{2} \mathrm{CO}_{2}\right\}_{2}\right]\right.$, an analogue of sodium aurothiomalate. The diagram was drawn with DIAMOND ${ }^{\circledR}$ (9). Colour code: Gold, orange; sulphur, yellow; oxygen, red; grey, carbon; hydrogen, green

mode of administration and therefore, biodistribution. The first class of compounds are generally polymeric, charged and water soluble. By contrast, the second class of compound is monomeric, neutral and lipophilic. Class I comprises (a) sodium aurothiomalate (e.g. Miocrin ${ }^{\circledR}$,

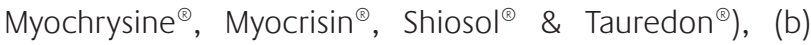

aurothioglucose (e.g. Aureotan ${ }^{\circledR}$, Auromyose ${ }^{\circledR}$, \& Solganol ${ }^{\circledR}$ ), (c) sodium aurothiopropanol sulphonate (Allochrysine Limière ${ }^{\circledR}$ ), and (d) sodium aurothiosulphate (Fosfocrisolo ${ }^{\circledR}$ \& Sanocrysin $\left.{ }^{\circledR}\right)$. The chemical formulations of the abovementioned compounds are illustrated in Figure 1 but, these are generally over-simplifications of the real structures, some of which are, in fact, not yet known.

A crystal structure determination is available for aurothiosulphate (8) as illustrated for the anion in Figure 2. This monomeric structure demonstrates the propensity of gold to exist in linear coordination geometries defined by two sulphur atoms and in this way gives a clue to the structures of the other gold drugs. Whereas there are two potential sulphur donor atoms in aurothiosulphate, the ratio of gold to sulphur in the remaining Class I compounds is 1:1 so that in order to achieve analogous S-Au-S entities, the sulphur atoms in these structures must be bidentate and bridging. Evidence in support of this conclusion has been provided recently by the crystal structure determination of the aurothiomalate anion in $\left[\mathrm{Na}_{2} \mathrm{Cs}(\mathrm{H})\right][\{\mathrm{Au}$ ( $\left.\mathrm{SCH}\left(\mathrm{CO}_{2}\right) \mathrm{CH}_{2} \mathrm{CO}_{2}\right\}_{2}$ ] (10). While this does not correspond to the medicinal formulation in terms of the cations, the polymeric array is consistent with expectation. As shown in Figure 3, the gold atoms exist in linear geometries defined by two sulphur atoms with this mode of association leading to the formation of helical chains (with four-fold symmetry). By contrast to the structural ambiguity associated with the Class I compounds, the precise geometric structure is known for the sole member of the Class II drugs, i.e. triethylphosphinegold(I) tetraacetylatedthioglucose, commonly known as auranofin $\left(\right.$ Aktil $^{\oplus}$, Crisinor $^{\oplus}$, Crisofin ${ }^{\circledR}$ \&

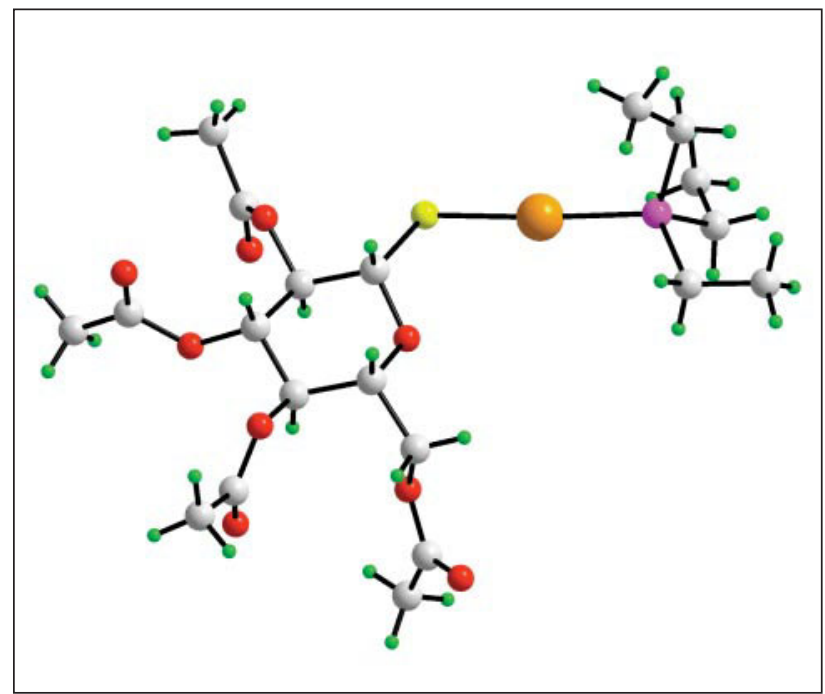

Figure 4

The molecular structure of auranofin as determined by X-ray crystallography. The diagram was drawn with DIAMOND ${ }^{\circledR}$ (9). Colour code: Gold, orange; sulphur, yellow; phosphorus, pink; oxygen, red; grey, carbon; hydrogen, green 
Ridaura $^{\circledR}$ ); structure (e) in Figure 1. The molecular structure of auranofin as determined by X-ray crystallography (11) is shown in Figure 4. The familiar linear coordination geometry about gold is again featured. However, in this case only one of the donor atoms is a sulphur atom as the other site is occupied by a phosphorus atom derived from the triethylphosphine ligand.

The above discussion may be summarised along the following lines. RA is not a well understood disease that may be treated but, not cured, by a variety of drugs, including gold-based drugs, the structures for which are, generally, not known. In the same way, the mechanism of action of golddrugs is not well understood. The following is a brief (and simplified) précis of the possible metabolic pathways of the Class I and II compounds.

As indicated above, owing to their chemical and physical properties, Class I and || gold-drugs are administered differently. The two most widely used Class I compounds in chrysotherapy are sodium aurothiomalate and aurothioglucose. These are typically administered by weekly intramuscular injections of suspensions containing 50 mg of 'gold', after a two week induction period in which smaller quantities of gold are delivered in order to monitor for sideeffects. Benefits from chrysotherapy using Class I drugs are usually manifest after three to six months. Treatment can result in a decrease in the painful swellings associated with inflammation of joint linings and can prevent destruction of bone and cartilage. Side-effects may appear immediately upon treatment up to months after treatment has stopped and may include dermatitis, discolouration of the skin, metallic taste, diarrhoea, kidney and bone marrow problems. The mode of administration of the Class I drugs indicate that the drugs are delivered to the vascular system. This is rich in the blood protein albumin and it is thought that the gold drug undergoes a substitution reaction so that one of the thiomalate ligands is replaced by an albumin molecule. Subsequent metabolism may involve the substitution of the second thiolate ligand of the drug by another albumin molecule resulting in the original gold atom existing within a ball of protein that is presumably carried to the site of inflammation. Once at the diseased site, the gold may be extracted by cyanide (a metabolite of thiocyanate and generated at sites of inflammation) to form a small anionic species that is known to have a very high stability constant, i.e. $\left[\mathrm{Au}(\mathrm{CN})_{2}\right]^{-}$. It may be that this is the active species. A similar mechanism of action may be envisaged for auranofin.

Auranofin is administered orally, typically in capsules containing 3- 6 mg 'gold', on a daily basis. This means that auranofin is delivered to the gastrointestinal tract from which approximately $25-30 \%$ of the gold is absorbed implying that only small amounts of gold are retained by the body. It appears that the gold-sulfur bond is readily cleaved and albumin coordinated in its stead. The second step in the metabolic pathway may involve coordination of another thiol such as albumin or glutathione, reduction of the coordinated albumin molecule and oxidation of the triethylphosphine to triethylphosphineoxide. In this way, a gold protein complex is generated as for the Class I drugs. The interested reader is directed to reviews discussing the biochemistry of gold drugs $(3 \mathrm{~b}, 12,13)$. The obvious conclusion of what is known about the metabolic pathways of both Class I and II drugs is they the administered drugs rapidly undergo substitution reactions of one sort or another and therefore, gold drugs must be regarded as prodrugs.

Given the above, there is enormous scope for drug development for the treatment of RA. For gold-based drugs, outside his own work in this area, that has generated gold compounds of significantly greater efficacy and reduced toxicity (e.g. 14, 15), the author is not aware of other studies directed towards developing new gold-based drugs in this context. Nevertheless, there is great interest in developing gold compounds as other therapies.

\section{Other Therapeutic Potential of Gold Compounds}

As discussed above, the aetiology of RA is not known and indeed many causes of the disease have been cited such as induction by a simple mechanical knock. Possible biological causes include malfunction in the immune system, viral and bacterial infections. As such, gold compounds have been evaluated for activity against other diseases owing to the different possible causes of RA, as summarised below.

In the desperate quest to seek a cure for AIDS, some gold drugs have been evaluated for activity against human immunodeficiency virus (HIV). Early results suggest that there might be some inhibition of HIV exhibited by gold compounds such as sodium aurothiomalate and aurothioglucose (5, 16 19). Some gold compounds used in the treatment of RA have also demonstrated effectiveness against severe bronchial asthma owing to their immunosuppressant properties (7, 20, 21). Recent reviews describing new/different agents for treating asthmatics suggest that gold-therapy might be limited to severe cases (22 - 24), such as chronic corticosteroid-dependent asthma, i.e. as second-line agents as for the treatment of RA by DMARDS. Phosphinegold(I) thiolates have also been evaluated for their activity against parasitic diseases such as malaria (6) and Chagas disease (25). However, in terms of drug development, most attention has been devoted to the investigation of the anti-tumour potential of gold compounds (4) and this forms the focus of the remaining part of this review. 


\section{Gold Compounds as Anti-Tumour Agents}

There are several reasons as to why gold compounds should be investigated for their potential as anti-tumour agents, based on both the medical and chemical literature. First and foremost was the dual biological activity exhibited by molecules such as cyclophosphamide, 6-mercaptopurine and methotrexate. These known anti-tumour agents also display immunosuppressive and anti-inflammatory characteristics and hence, their evaluation against RA was undertaken. This, in a sense, establishes a connection between the two therapies, even if tenuous. Allied to this were the results of a long-term study of patients undergoing chrysotherapy (26). This study suggested that such patients showed no enhanced risk or even reduced risk of malignant diseases, a result that could be correlated with the administration of gold drugs. Other factors that suggest a study of the anti-tumour potential of gold compounds might be worthwhile relates to the fact that gold in the +3 oxidation state has the same electronic configuration and structural characteristics as the World's most widely-used anti-cancer drug, cisplatin, i.e. $\left(\mathrm{NH}_{3}\right)_{2} \mathrm{PtCl}_{2}$. The other main reason relates to the observation, mentioned above, that gold drugs are in fact prodrugs. In this regard, gold may be thought of as a platform designed to deliver biologically active molecules, i.e. drugs, to the body. Coordination of biomolecules as such would obviously alter the normal metabolic pathways, allow for slow-release mechanisms, etc. and may lead to greater efficacy and reduced toxic side-effects associated with organic molecules when administered alone. Whatever the reasons for studying the anti-tumour potential of gold compounds, the following discussion will demonstrate that gold compounds display exciting cytotoxicity profiles, based on in vitro assays, leading to interesting anti-tumour activities, based on animal (in vivo) studies.

The first comprehensive studies of the anti-tumour potential of gold compounds, including gold drugs, were published in the mid- to late-1980's [e.g. 27]. The conclusions of these studies still have relevance today and are worth restating. As a general principle to guide researchers in this area, it was reported that gold compounds that were active in vitro were not necessarily active in vivo, akin to other studies, but, importantly, compounds that were inactive in vitro were unlikely to be active in vivo. Hence, a rapid screening of anti-tumour potential for gold compounds can be achieved based on in vitro studies. A key outcome of this early work was the conclusion that gold was essential for elevated potency. These studies also showed that the most promising class of compounds, at least containing gold(I), were the phosphinegold $(I)$ thiolates, i.e. related to the structure of auranofin (Figure 4). Thus, gold(I) thiolates related to the Class I anti-arthritic drugs were generally inactive as were non-phosphine compounds and non-thiolate compounds. In a sense, this was the first structure activity relationship that was established: active compounds feature the P-Au-S arrangement as found in the Class II RA drug, auranofin. A subsequent study extended the range of gold compounds to include those containing bidentate phosphines $(28,29)$.

A particularly interesting observation was the rearrangement, in solution and in biological media, of some of the diphosphine compounds to yield a comparatively rare coordination geometry, for gold(I), based on a tetrahedral arrangement of four phosphorus donor atoms around gold as illustrated in Figure 5 (30). These turned out to be a particularly potent class of compounds but clinical trials were not pursued owing to the acute toxicity associated with them. However, subsequent studies have focused on chemically modifying the aromatic substituents, such as to form n-pyridyl analogues, so as to moderate the lipophilicity of the compounds and to emphasise their apparent anti-mitochondrial activity; a recent review summarises in more detail advances in this research endeavour (31). Given the indications of the initial studies examining the anti-tumour potential of gold compounds, i.e. the potency of auranofin and analogues, it is perhaps not surprising that the most significant effort has been devoted to investigating the potential of auranofin analogues.

Given the fact that the clinically used gold compounds are prodrugs, as mentioned a few times above, a logical extension of earlier work was to couple phosphinegold(I) species to biologically active thiols. This principle proved fruitful in screening for anti-arthritic activity where thiols such as 6-mercaptopurine and 6-thioguanine were utilised (14, 15); see above. These thiols are also known to possess anticancer activity against human leukaemia and a study of the potential anti-tumour activity of their phosphinegold(I) compounds ensued. The prototype structure, i.e. of triphenylphosphinegold(I) 6-mercaptopurinate, abbreviated as $\mathrm{Ph}_{3} \mathrm{PAu}(6-\mathrm{MP})$, for these compounds is shown in Figure 6 (14). Several important conclusions were gained from the study of these compounds (31-34).

As concluded from the earlier studies, it is clear that the presence of the phosphinegold(I) entity enhances the potency of the biologically-active thiols. Thus, greater overall cytotoxicity was observed for the phosphinegold(I) thiolates compared with the free thiols in a range of human cancer cell lines. Further, the compounds showed greater potency than cisplatin in these in vitro models, surely the benchmark in metal-based anti-cancer treatment, as well as other standard drugs. Preliminary anti-tumour screening, i.e. in vivo was also conducted that showed that activity was maintained to a certain extent (32). The principle of coupling therapeutically important molecules to phosphinegold $(I)$ entities is quite clearly vindicated as may be seen from the data presented in Table 1 (33). The results of the in vitro assay against a 


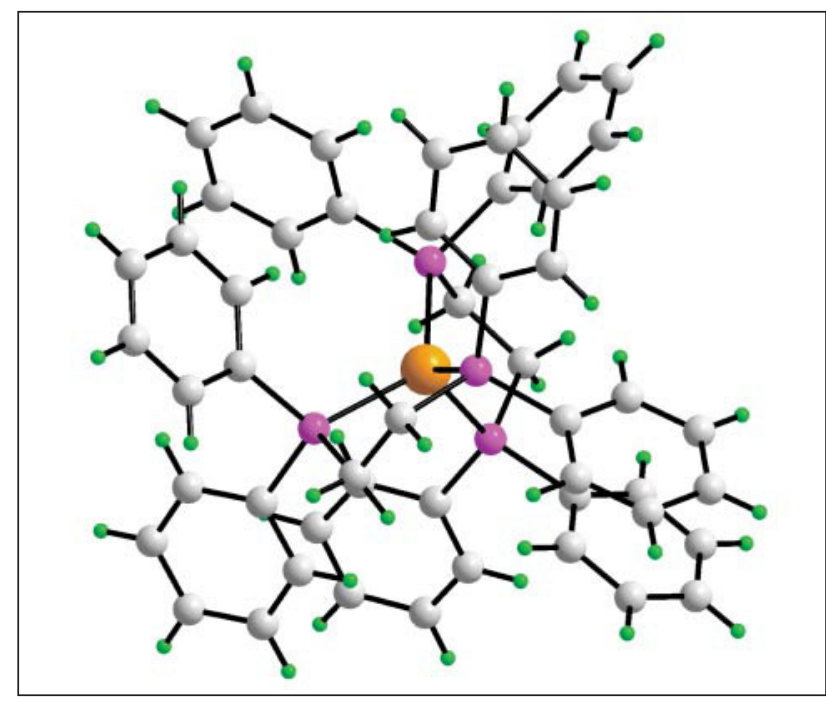

Figure 5

The molecular structure of the $\left[\mathrm{Au}(\mathrm{dppe})_{2}\right]^{+}$cation as determined by $X$-ray crystallography. The diagram was drawn with DIAMOND ${ }^{\circledR}$ (9). Colour code: Gold, orange; phosphorus, pink; grey, carbon; hydrogen, green

Table 1

Selected cytotoxicity and anti-tumour screening results

\begin{tabular}{|c|c|c|}
\hline Compound & $\mathrm{IC}_{50}^{\mathrm{a}}$ & Growth inhibition ${ }^{b}$ \\
\hline 6-MPH & 3.3 & no effect \\
\hline $\mathrm{Ph}_{3} \mathrm{PAuCl}^{\mathrm{c}}$ & 0.14 & no effect \\
\hline $\mathrm{Ph}_{3} \mathrm{PAu}(6-\mathrm{MP})^{\mathrm{d}}$ & 0.05 & $60 \%$ \\
\hline cisplatin & 6.7 & $100 \%$ \\
\hline \multicolumn{3}{|c|}{$\begin{array}{l}\text { a I } C_{50} \text { value in } \mu M \text { units - the amount of compound required to inhibit } \\
50 \% \text { growth of } L 1210 / D D P \text { leukaemia cancer cells; the smaller the } \\
\text { value, the greater the potency }\end{array}$} \\
\hline \multicolumn{3}{|c|}{$\begin{array}{l}\text { b Percentage growth inhibition of PC6 tumours grown in female } \\
\text { Balb/C mice; the greater the value, the more effective the } \\
\text { compound }\end{array}$} \\
\hline \multicolumn{3}{|c|}{$\begin{array}{l}\text { c 6-MPH is 6-mercaptopurine; } P h_{3} P \text { is triphenylphosphine } \\
d \text { The molecular structure of this compound is illustrated in Figure } 6\end{array}$} \\
\hline
\end{tabular}

cisplatin-resistant leukaemia cell line (L1210/DDP) demonstrate i) all compounds are more potent in this cell line than cisplatin (a result consistent with the known antileukaemia activity of 6-MPH) and ii) while each of the $\mathrm{Ph}_{3} \mathrm{PAuCl}$ and 6-mercaptopurine (6-MPH) compounds show potency, the most potent compound is the one that combines both phosphinegold $(I)$ and the anion derived from 6-MPH. Anti-tumour screening against PC6 plasmacytoma (a cisplatin-sensitive murine cell line) showed that the $\mathrm{Ph}_{3} \mathrm{PAu}(6$ MP) significantly reduced the size of the tumour but not as effectively as cisplatin. Importantly, this study showed that promising in vitro results were maintained in an animal model; it is noted that the relevance of animal tumours to human tumours is debatable. Interest in these compounds was sufficient to warrant a detailed examination of cytotoxicity by the National Cancer Institute (U.S.A.) against a panel of 60 cancer cell lines. Perhaps not surprising given the known activity against leukaemia displayed by the thiols, 6mercaptopurine and 6-thioguanine, sub-panel selectivity against leukaemia cell lines was observed. For the limited series of phosphines examined when the thiolate derived from 6-mercaptopurine constant, i.e. for the series of compounds $\mathrm{R}_{3} \mathrm{PAu}(6-\mathrm{MP})$, a structure/activity relationship was established in that the order of cytotoxicity was $R=$ cyclohexyl > ethyl > phenyl $(33,34)$. The apparent selectivity for a particular cancer cell line, in this case, leukaemia, was particularly encouraging and this theme is explored further for the next series of compounds that were examined.

As part of an on-going attempt to alter the solubility characteristics of phosphinegold(I) thiolates, an isomeric series of n-mercaptobenzoates for $n=2-$, 3- and 4-, were studied $(36,37)$. For compounds of the general formula $\mathrm{R}_{3} \mathrm{PAu}\left(\mathrm{SC}_{6} \mathrm{H}_{4}-\right.$ $\left.\mathrm{CO}_{2} \mathrm{H}-n\right)$, those with $n=2$ were more cytotoxic than the $n=3$ and $n=4$ analogues. More exciting was the observation that for the $n=2$ series, several of the compounds displayed selective activity against the A498 (renal cancer) and H226 (non-small cell lung cancer) cell lines, exhibited cytotoxicities far better than cisplatin and were more potent or had comparable potency to established anti-cancer compounds such as doxorubicin and methotrexate $(36,37)$. On-going studies are focusing on determining the maximum tolerated doses and anti-tumour activities of these compounds; these results will be published in due course but are most encouraging. A recent review detailing the work emanating from our own laboratories has just been published (38).

Thus far, the focus of discussion in this overview has been upon gold(I) compounds. However, an area of research gaining prominence, based on recent publications, features gold(III) compounds. Gold(III) would normally be regarded as oxidising and this property could be exacerbated in the reducing mammalian environment. However, judicious choice of donor atoms in the ligand donor set can impart stability to the higher oxidation state; gold(III) is regarded as an "harder" acid than gold(I) and hence, is more likely to form stable compounds with donor atoms such as nitrogen and oxygen whereas gold(I) exhibits a distinct preference for sulphur and phosphorus donor atoms.

The gold(III) compounds investigated for potential antitumour activity are inevitably four-coordinate and feature square planar geometries, as found for cisplatin so that, perhaps, a similar mechanism of action, i.e. interaction with DNA and disruption of normal cellular processes, may be assumed for these compounds. In this connection, evidence has been provided showing that some species bind DNA (39). There are basically three classes of gold(III) compounds receiving serious attention at present. The three classes are i) 


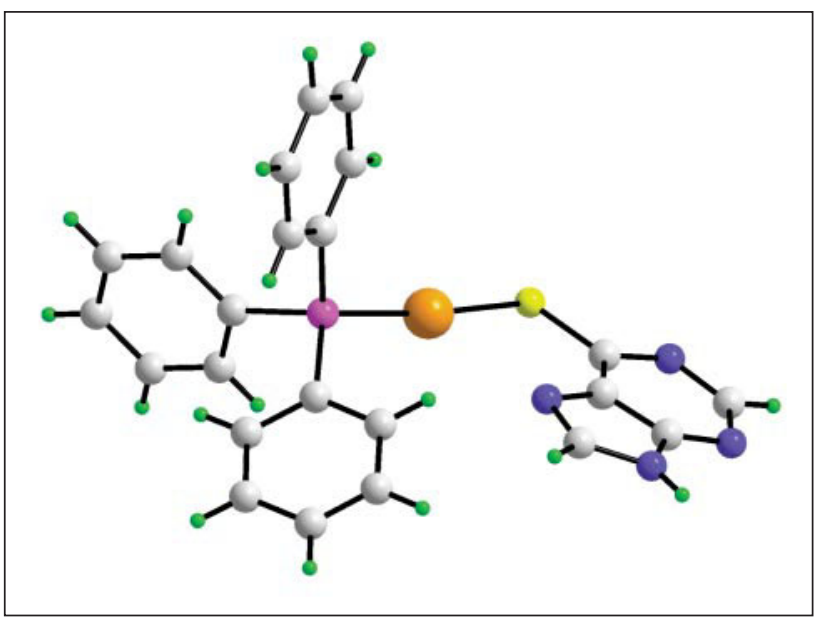

Figure 6

The molecular structure of triphenylphosphinegold(I) 6mercaptopurinate as determined by X-ray crystallography. The diagram was drawn with DIAMOND ${ }^{\circledR}$ (9). Colour code: Gold, orange; sulphur, yellow; phosphorus, pink; blue, nitrogen; grey, carbon; hydrogen, green

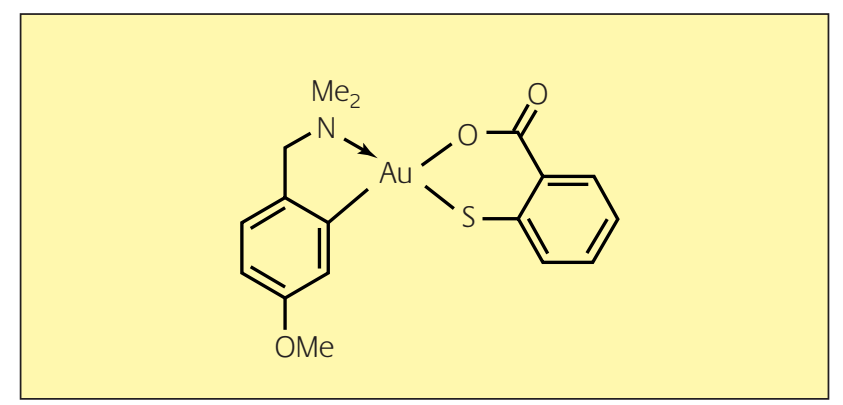

Figure 7

The proposed chemical structure of a gold(III) compound, [\{p$\mathrm{MeOC}_{6} \mathrm{H}_{3} \mathrm{CH}_{2} \mathrm{NMe}_{2}$ \} Au $\left.\left(\mathrm{SC}_{6} \mathrm{H}_{4} \mathrm{COO}_{2}-2\right)\right]$

those incorporating biologically active species such as streptonigrin (40); ii) compounds with imine (pyridine-type nitrogen) donor atoms (41); and iii) organogold compounds, i.e. containing Au-C bonds (42). Whereas the overwhelming majority of gold(III) compounds investigated thus far contain ligand donors derived from one or some combination of chloride, nitrogen, oxygen, and carbon. Perhaps it is significant that arguably the compound showing the most promise contains a sulphur donor atom derived from 2mercaptobenzoate, See Figure 7 (42b), as found in their phosphinegold(I) analogues discussed above.

\section{Conclusions and Outlook}

The above discussion describes some of the current medicinal uses of gold compounds, namely in the remediation of the symptoms associated with rheumatoid arthritis. Other therapies based on gold may include their use as anti- parasitic, -HIV and -asthma agents. Arguably the most active research in medicinal gold chemistry involves the development of novel anti-tumour agents. Three areas in particular attract significant attention although there are other strategies worth pursuing not mentioned above such as for example heterometallic systems (4). The three areas are i) developing tetrahedrally coordinated gold(I) compounds that may target mitochrondria, ii) generating phosphinegold(I) thiolates where the gold is employed as a carrier of biologically-active substrates, and iii) forming square planar gold(III) compounds that may also target DNA. In fact, in terms of recent publications, gold(III) species are receiving the most attention such as gold(III) porphyrin (43) and aminoquinoline (44) derivatives. An interesting development involves the three-coordinate gold(I) species with both mono- and bi-dentate phosphine ligands (45). The foregoing shows that i) there is still considerable interest, at least amongst the academic community, in developing gold compounds for anti-tumour screening, and ii) the variations in gold compounds are far from exhausted with novel compounds waiting to be generated.

In developing new anti-tumour agents, researchers should be mindful of several factors required for the successful adoption of new therapeutic agents such as the need to target cisplatin-resistant cancer cell lines, to reduce deleterious side-effects associated with chemotherapy and, related to this, to target specific biological sites, i.e. to increase the selectivity of the anti-cancer agents. However, too strict an adherence to these principles might preclude serendipitous discoveries, keeping in mind that the biological activity of cisplatin was noted by keen experimental observation rather than rational drug design. In terms of antitumour screening, the most reliable biological evaluation will be obtained from in vivo models of human cancer cell lines but, only after mandatory in vitro cytotoxicity screening.

\section{Acknowledgements}

On-going research at the National University of Singapore is supported by the National University of Singapore ( $R$ 143000-213-112) and the Biomedical Research Council (Singapore).

\section{About the Author}

Edward R.T. Tiekink is a graduate of The University of Melbourne (Ph.D., 1986) from where he moved to The University of Adelaide, first as a Research Associate and later, joining the academic staff in 1989. It was here that an 
interest in medicinal gold chemistry developed. In July 2001, the author moved to the National University of Singapore where he pursues research interests revolving around X-ray crystallography and in particular upon the understanding and controlling of the influence of crystal structure on molecular geometries, especially of main group element compounds. These interests are reflected in his membership of the Editorial Boards of Acta Crystallographica E (Founding CoEditor), Bioinorganic Chemistry \& Applications, Applied Organometallic Chemistry (Section Co-Editor) and Zeitschrift für Kristallographie (Co-Editor). He is the author/co-author of in excess of 850 papers, reviews, crystallographic reports and patents.

\section{References}

1 W.F. Kean, F. Forestier, Y. Kassam, W.W Buchanan, P.J. Rooney, Semin. Arthritis Rheum. 1985, 14, 180

2 H. Zhao, Y. Ning, Gold Bull. 2001, 34, 24

3 e.g. (a) A.J. Lewis and D.T. Walz, Prog. Med. Chem. 1982, 19, 1; (b) E.R.T. Tiekink, M.W. Whitehouse, in G. Berthon (Ed) Handbook of MetalLigand Interactions in Biological Fluids. Vol. 2. Marcel Dekker (New York), 1995, 1266

4 e.g. E.R.T. Tiekink, Crit. Rev. Oncol. Hematol. 2002, 42, 225

5 e.g. T. Okada, B.K. Patterson, S.-Q. Ye, M.E. Gurney, Virology, 1993, 192, 631

6 M. Navarro, H. Pérez, R.A. Sánchez-Delgado, J. Med. Chem. 1997, 40, 1937

7 M. Muranaka, T. Miyamoto, T. Shida, J. Kabe, S. Makino, H. Okumura, K. Takeda, S. Suzuki, Y. Horiuchi, Ann. Allergy, 1978, 40, 132

8 H. Ruben, A. Zalkin, M.O. Faltens, D.H. Templeton, Inorg. Chem. 1974, 13, 1836

9 DIAMOND, Visual Crystal Structure Information System, Version 2.1e, CRYSTAL IMPACT, Postfach 1251, D-53002 Bonn, Germany, 2002

10 R. Bau, J. Am. Chem. Soc. 1998, 120, 9380

11 D.T. Hill, B.M. Sutton, Cryst. Struct. Commun, 1980, 9, 679

12 C.F. Shaw III, Chem. Rev. 1999, 99, 2589

13 C.F. Shaw III, Topics Biol. Inorg. Chem. 1999, 2, 187

14 P.D. Cookson, E.R.T. Tiekink, M. W. Whitehouse, Aust. J. Chem. 1994, 47, 577

15 M.W. Whitehouse, P.D. Cookson, G. Siasios, E.R.T. Tiekink, Metal-Based Drugs, 1998, 5, 245

16 K. Tepperman, Y. Zhang, P.W. Roy, R. Floyd, Z. Zhao, J.G. Dorsey, R.C. Elder, Metal-Based Drugs, 1994, 1, 433

17 C.F. Shaw III, Uses of Inorganic Chemistry in Medicine, N. P. Farrell, (Ed), Royal Society of Chemistry, 1999, 26

18 K.E. Traber, H. Okamoto, C. Kurono, M. Baba, C. Saliou, T. Soji, L. Packer, T. Okamoto, Int. Immunol. 1999, 11, 143

19 K. Yamaguchi, H. Ushijima, M. Hisano, Y. Inoue, T. Shimamura, T. Hirano, E. G. W. Muller, Microbiol. Immunol. 2001, 45, 549
20 M. Adachi, H. Sato, T. Takahashi, Ther. Res. 1986, 5, 796; CAN 106:168349

21 A.C. Falcao, M.J. Rocha, A.M. Almeida, M.M. Caramona, J. Clin. Pharma. Ther. 2000, 25, 191

22 M.S. Dykewicz, Allerg. Asth. Proc. 2001, 22, 11

23 A.M. Vignola, P. Chanez, J. Bousquet, in Lung Biology in Health and Disease, 2001, 159, 575

24 A.J. Frew, M.J. Plummeridge, J. Allergy Clin. Immuno. 2001, 108, 3

25 M. Navarro, E.J. Cisneros-Fajardo, T. Lehmann, R.A. Sánchez-Delgardo, R. Atencio, P. Silva, R. Lira, J.A. Urbina, Inorg. Chem. 2001, 40, 6879

26 J.F. Fries, D. Bloch, P. Spitz, D.M. Mitchell, Am. J. Med. 1988, 85 (Suppl. 4A), 56

27 C.K. Mirabelli, R.K. Johnson, D. T. Hill, L. F. Faucette, G.R. Girard, G.Y. Kuo, C.-M. Sung, S.T. Crooke, J. Med. Chem. 1986, 29, 218

28 C.K. Mirabelli, B.D. Jensen, M.R. Mattern, C.-M. Sung, S.-M. Mong, D.T. Hill, S.W. Dean, P.S. Schein, R.K. Johnson, S.T. Crooke, Anticancer Drug Des. 1986, 1, 223

29 C.K. Mirabelli, D.T. Hill, L. F. Faucette, F.L. McCabe, G.R. Girard, D.B. Bryan, B.M. Sutton, J. O’Leary Bartus, S.T. Crooke, R.K. Johnson J. Med. Chem. 1987, 30, 2181

30 S.J. Berners-Price, M.A. Mazid, P.J. Sadler, J. Chem. Soc., Dalton Trans, 1984, 969

31 M.J. McKeage, L. Maharaj, S.J. Berners-Price, Coord. Chem. Rev. 2002, 232, 127

32 E.R.T. Tiekink, P.D. Cookson, B.M. Linahan, L.K. Webster, Metal-Based Drugs, 1994, 1, 299

33 L.K. Webster, S. Rainone, E. Horn, E.R.T. Tiekink, Metal-Based Drugs, 1996, 3, 63

34 D. Crump, G. Siasios, E.R.T. Tiekink, Metal-Based Drugs, 1999, 6, 361.

35 E.R.T. Tiekink, Metals Ions Biol. Med., 1996, 4, 693

36 D. de Vos, P. Clements, S.M. Pyke, D.R. Smyth and E.R.T. Tiekink, MetalBased Drugs, 1999, 6, 31

37 D. de Vos, D.R. Smyth, E.R.T. Tiekink, Metal-Based Drugs, 2001, 8, 303

38 E.R.T. Tiekink, Bioinorg. Chem. Appln, 2003, 1, 53

39 (a) S. Carotti, A. Guerri, T. Mazzei, L. Messori, E. Mini, P. Orioli, Inorg Chim Acta, 1998, 81, 90; (b) S. Carotti, G. Marcon, M. Marussich, T. Mazzei, L. Messori, E. Mini, P. Orioli, Chem.-Biol, Inter. 2000, 125, 29

40 e.g. A. Moustatih, A. Garnier-Suillerot, J. Med. Chem. 1989, 32, 1426.

41 e.g. L. Messori, F. Abbate, G. Marcon, P. Orioli, M. Fontani, E. Mini, T. Mazzei, S. Carotti, T. O’Connell, P. Zanello, J. Med. Chem. 2000, 43, 3541

42 e.g. (a) R.V. Parish, Metal-Based Drugs, 1999, 6, 271; (b) M.B. Dinger, W. Henderson, J. Organomet. Chem. 1998, 560, 233

43 C.-M. Che, R.W.-Y. Sun, W.-Y. Yu, C.-B. Ko, N. Zhu, H. Sun, Chem Commun, 2003, 1718

44 T. Yang, C. Tu, J. Zhang, L. Liu, X. Zhang, Q. Liu, J. Ding, Q. Xu, Z. Guo, Dalton Trans, 2003, 3419

45 F. Caruso, M. Rossi, J. Tanski, C. Pettinari, F. Marchetti, J. Med. Chem. 2003, 46, 1737 